\title{
Unexpectedly low seroprevalence of toxoplasmosis in South Africa
}

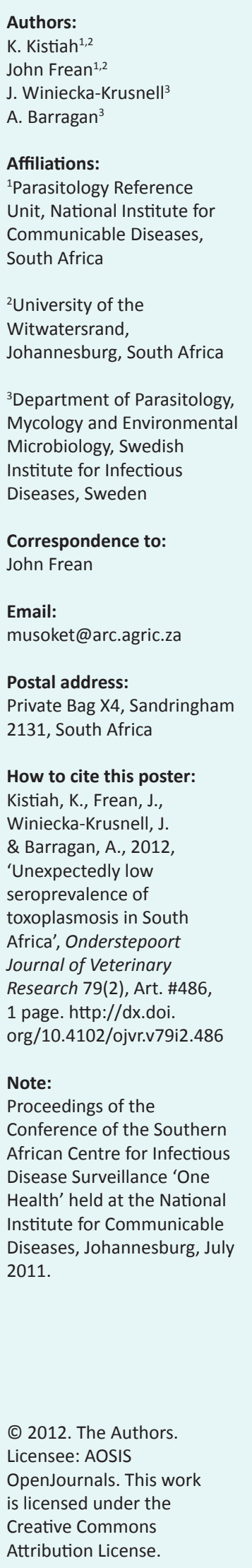

Toxoplasmosis is an infection of warm-blooded vertebrates caused by the obligate intracellular protozoan parasite, Toxoplasma gondii. It is one of the most common parasitic diseases of humans, infecting approximately one-third of the world's population. In persons with advanced HIV, toxoplasmosis represents a major opportunistic infection of the central nervous system. Approximately two-thirds of all people living with HIV live in sub-Saharan Africa. In areas such as this, toxoplasmosis could theoretically pose a huge threat. There is little known about T. gondii prevalence in humans in Africa. Geographically, prevalences vary widely on this continent, as observed in other parts of the world. There is limited historical information about the disease in South Africa. More knowledge is needed at a regional level about the risk of toxoplasmosis, diagnostic issues, and measures to reduce the risk to susceptible persons. The seroprevalence of T. gondii in selected populations, namely HIV-positive and HIV-negative individuals, and a more general sample biased towards pregnant women, was therefore investigated and found to be $9.8 \%(37 / 376), 12.8 \%(48 / 376)$ and $6.4 \%(32 / 497)$ respectively. Compared with historical data from South Africa, the prevalence has decreased substantially; however, the incidence of clinical disease is unknown, despite the very high burden of HIV and AIDS cases (5.9 million and 0.7 million, respectively in 2009). This study provided information relating to the diagnosis and current seroprevalence of T. gondii in South Africa. Many questions still remain to be answered however, to fully understand the impact of this parasite on the country's population. 